\title{
Prevention of bleeding in patients with haemophilia undergoing surgery
}

\section{Anne Wareing}

There is little evidence on which to define the best treatment for the prevention of bleeding in people with congenital bleeding disorders undergoing surgery. As a result, treatment regimens are frequently based on local protocols developed by expert clinicians and information from uncontrolled observational studies. This article summarises the Cochrane Cystic Fibrosis and Genetic Disorders Group systematic review on 'Treatment for preventing bleeding in people with haemophilia or other congenital bleeding disorders (CBDs) undergoing surgery'.

\section{Keywords: surgery, bleeding, haemophilia}

Why conduct this review?

Currently all types of surgeries, major and minor, may be carried out on patients with CBDs, provided that haemostasis teams are able to provide adequate haemostasis coverage throughout the periods of bleeding risk and until wound healing is complete [1]. Whilst there are guidelines on surgery available from national scientific panels and expert panels, the optimal duration and target levels of haemostatic treatment have not yet been definitively established.

Furthermore, evidence suggests there are significant issues related to surgery which merit further consideration, such as the possible risk of inhibitor development with continuous infusion and the use of thromboprophylaxis in major orthopaedic surgery.

The clinical question

To assess the effectiveness and safety of different haemostatic regimens, i.e. type, dose and duration, modality of administration and target haemostatic levels, administered in people with a CBD for preventing bleeding complications during and after surgical procedures.

Study selection and outcome measures

Population: Studies were included from both adult and children with congenital bleeding disorders of any severity and included those with inhibitors.

Intervention: Any haemostatic treatment regimen.

Comparison: No treatment or another active regimen.

Outcomes: Primary outcome measures were mortality and

Anne Wareing, Clinical Nurse Specialist, Department of Haemostasis, St George's Univerity Hospital NHS Foundation Trust, Blackshaw Road, London SW17 0QT, UK. Email: anne.wareing anhs.net

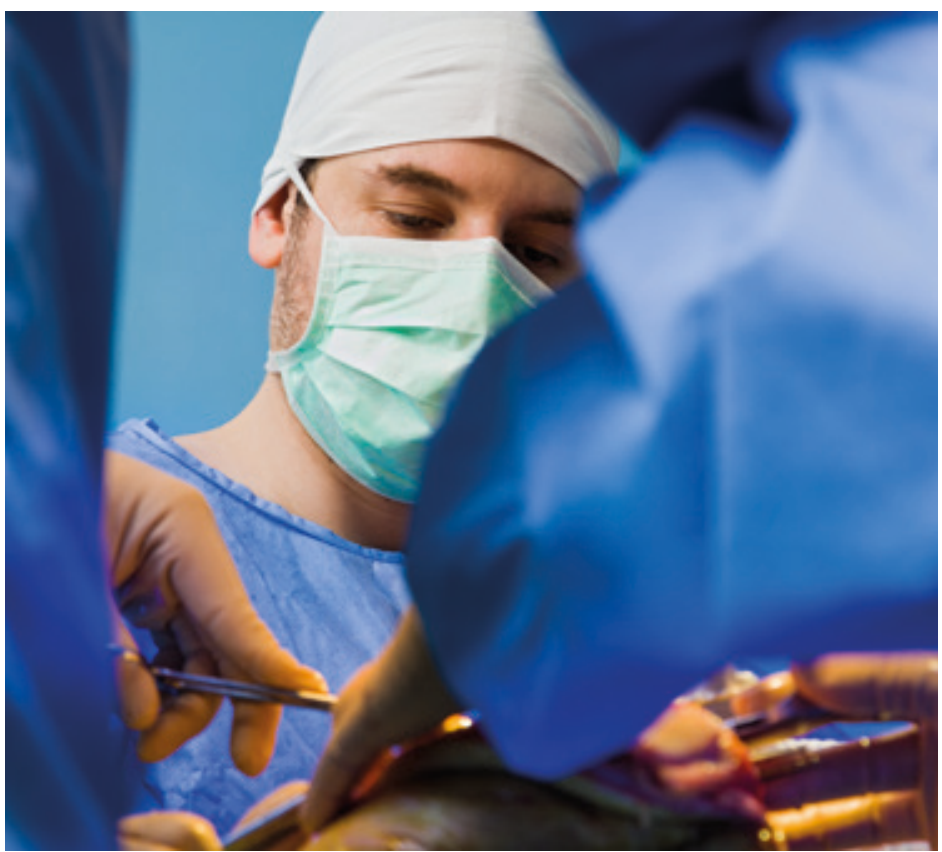

OShutterstock Inc.

blood loss (defined by transfusion requirements during and after surgery.) and the need for further intervention (i.e. further surgeries).

Studies: Randomised and quasi-randomised controlled trials. Of the 16 identified trials, four (112 participants) were eligible for inclusion

Search strategy and data collection

Studies were identified from the Cystic Fibrosis and Genetic Disorders Group's Coagulopathies Trials Register. This register is compiled from electronic searches of the Cochrane Central Register of Controlled Trials, weekly searches of MEDLINE and prospective manual searching of Haemophilia. Searches were also conducted on abstracts from conferences and meetings of the European Haemophilia Association, the American Society of Haematology, the British Society for Haematology, the International Society of Haemostasis and Thrombosis and the International Congress of World Federation of Hemophilia.

In terms of inclusion criteria, there were no restrictions on date, language, publication type or status of study.

Results

Eligible trials related to two specific clinical settings and therapeutic interventions, namely dental interventions [2, 3] 
and surgery on patients with inhibitors [4,5].

The dental studies reported on 59 participants and 63 procedures, and were placebo controlled. Patients with haemophilia A or B undergoing dental extractions were given factor replacement with or without antifibrinolytic agents. Mortality was not specifically addressed in either of the trials; however the use of antifibrinolytic agents reduced blood loss and requirement of post-operative replacement treatment compared with placebo.

The patients with haemophilia A or B with inhibitors undergoing surgery were treated with different regimens of rFVIla for haemostatic coverage. 53 participants were considered, 33 of whom underwent major surgery. Shapiro's trial randomised patients to receive either $35 \mu \mathrm{g} / \mathrm{kg}$ or high dose $90 \mu \mathrm{g} / \mathrm{kg}$ of recombinant factor VIla preoperatively and at timed doses postoperatively [5]. The results revealed a higher postoperative haemostatic efficacy when a highdose $(90 \mu \mathrm{g} / \mathrm{kg})$ regimen of $\mathrm{rFVlla}$ was used, resulting in shorter duration of treatment and fewer injections, but a similar total dose of rFVIla administered and similar safety. Pruthi's trial compared different doses of rfVIla $(50 \mu \mathrm{g} / \mathrm{kg}$ and $90 \mu \mathrm{g} / \mathrm{kg}$ ) as well as different modalities of delivery: bolus versus continuous treatment [4]. There was no difference in efficacy, duration of treatment and safety between bolus infusion and continuous infusion of rFVIla.

\section{Conclusions}

The trials in this review provide information relating to two specific situations in people with congenital bleeding disorders undergoing surgery. However, on the whole, there is not enough evidence to define the best treatment for the various types of disease and types of surgery. Consequently, treatment regimens are often based on local protocols developed by expert clinicians and information from uncontrolled observational studies.

Several reasons are provided for the lack of robust data:

- The rarity of the conditions makes it difficult to undertake trials with adequate sample sizes.

- Limitations on the availability and safety of replacement products after the transmission of HIV and hepatitis in contaminated blood products made surgery difficult in the mid-1980s.

- Finally, it is argued that excellent clinical outcomes have been achieved in countries where factor concentrates are readily available, reducing the need for rigorous trials to consider optimal treatment regimens in terms of dosing, target factor levels and duration of therapy.

It is also noted that international guidelines, such as those published by the World Federation of Hemophilia [6], do not address the economic issues relating to surgical care of patients of CBDs, specifically cost-effective use of treatment.

\section{Future studies}

As with other rare diseases, this review highlights the difficulties of providing rigorous evidence in CBDs. It also emphasises the need for a broader consensus on outcome measures to facilitate comparison and pooling of data for future studies.

As readers of this journal will be aware, there are currently a plethora of clinical trials being undertaken, including trials of gene therapies, subcutaneous and longeracting products. It is anticipated that these products will substantially improve quality of life and mortality of patients with CBDs more generally. However, the focus on product development has possibly detracted from other research that will improve the surgical management of patients with CBDs. Nevertheless, as care improves it is likely that patients with CBDs will undergo more surgical procedures and a stronger evidence base will be needed to maintain standards of care and reduce the risk of complications.

\section{Disclosures}

The author has advised no interests that might be perceived as posing a conflict or bias.

This is an Open Access article distributed under the terms of the Creative Commons Attribution License (http:// creativecommons.org/licenses/by/2.0), which permits unrestricted use, distribution, and reproduction in any medium, provided the original work is properly cited.

\section{References}

1. Coppola A, Windyga J, Tufano A, Yeung C, Di Minno MND. Treatment for preventing bleeding in people with haemophilia or other congenital bleeding disorders undergoing surgery. Cochrane Database of Systematic Reviews 2015;2:CD009961. doi: 10.1002/14651858.CD009961.pub2.

2. Forbes CD, Barr RD, Reid G et al. Tranexamic acid in control of haemorrhage after dental extraction in haemophilia and Christmas disease. Br Med J 1972;2(5809):311-3.

3. Walsh PN, Rizza CR, Matthews JM, et al. Epsilon-Aminocaproic acid therapy for dental extractions in haemophilia and Christmas disease: a double blind controlled trial. Br J Haematol 1971;20(5):463-75.

4. Pruthi RK, Mathew P, Valentino LA, et al. Haemostatic efficacy and safety of bolus and continuous infusion of recombinant factor VIla are comparable in haemophilia patients with inhibitors undergoing major surgery. Results from an open-label, randomized, multicenter trial. Thrombosis and Haemostasis 2007;98(4):726-32.

5. Shapiro AD, Gilchrist GS, Hoots WK, Cooper HA, Gastineau DA. Prospective, randomised trial of two doses of rFVIla (NovoSeven) in haemophilia patients with inhibitors undergoing surgery. Thrombosis and Haemostasis 1998;80(5):773-8.

6. Srivastava A, Brewer AK, Mauser-Bunschoten P, et al. Guidelines for the management of haemophilia. Haemophilia. 2013;19(1):e1-47.

\section{Further reading}

Higgins, J.P. and Green, S. eds., (2008). Cochrane handbook for systematic reviews of interventions (Vol. 5). Chichester: Wiley-Blackwell. 